\title{
A New Genetic Algorithm Applied to Multi-Objectives Optimal of Upgrading Infrastructure in NGWN
}

\author{
Dac-Nhuong Le ${ }^{1}$, Nhu Gia Nguyen ${ }^{2}$, Dac Binh $\mathrm{Ha}^{2}$, Vinh Trong Le \\ ${ }^{1}$ Faculty of Information Technology, Haiphong University, Haiphong, Vietnam \\ ${ }^{2}$ Duytan University, Danang, Vietnam \\ ${ }^{3}$ Hanoi University of Science, Vietnam National University, Hanoi, Vietnam \\ Email: Nhuongld@hus.edu.vn, Nguyengianhu@duytan.edu.vn, hadacbinh@duytan.edu.vn, Vinhlt@vnu.edu.vn
}

Received June, 2013

\begin{abstract}
A problem of upgrading to the Next Generation Wireless Network (NGWN) is backward compatibility with pre-existing networks, the cost and operational benefit of gradually enhancing networks, by replacing, upgrading and installing new wireless network infrastructure elements that can accommodate both voice and data demand. In this paper, we propose a new genetic algorithm has double population to solve Multi-Objectives Optimal of Upgrading Infrastructure (MOOUI) problem in NGWN. We modeling network topology for MOOUI problem has two levels in which mobile users are sources and both base stations and base station controllers are concentrators. Our objective function is the sources to concentrators connectivity cost as well as the cost of the installation, connection, replacement, and capacity upgrade of infrastructure equipment. We generate two populations satisfy constraints and combine them to build solutions and evaluate the performance of my algorithm with data randomly generated. Numerical results show that our algorithm is a promising approach to solve this problem.
\end{abstract}

Keywords: Multi-Objectives Optimal; Next Generation Wireless Network; Network Design; Capacity Planning; Genetic Algorithm; Two-populations

\section{Introduction}

The Next Generation Wireless Networks (NGWNs) are expected to provide high data rate and optimized quality of service to multimedia and real-time applications over the Internet Protocol networks to anybody, anywhere, and anytime. The wireless network infrastructure consists of equipment required by mobile network operators to enable mobile telephony calls or to connect fix subscribers by radio technology. The interacting layers architecture of next generation wireless network is shown in Figure 1.

The PSTN-cloud covers all network elements to make a standard telephone call, while the data network cloud includes the Internet, Intranets, and other IP based networks [1]. The architectural building blocks enabling mobile telephony are:

- The core network: comprised of the mobile switching centers (MSC), the packet data serving nodes (PDSN), and home agents (HA), and

- $\quad$ The base station subsystem (BSS) also known as the radio access network, consisting of base station controllers (BSC), base transceiver stations (BS), and mobile stations (MS).

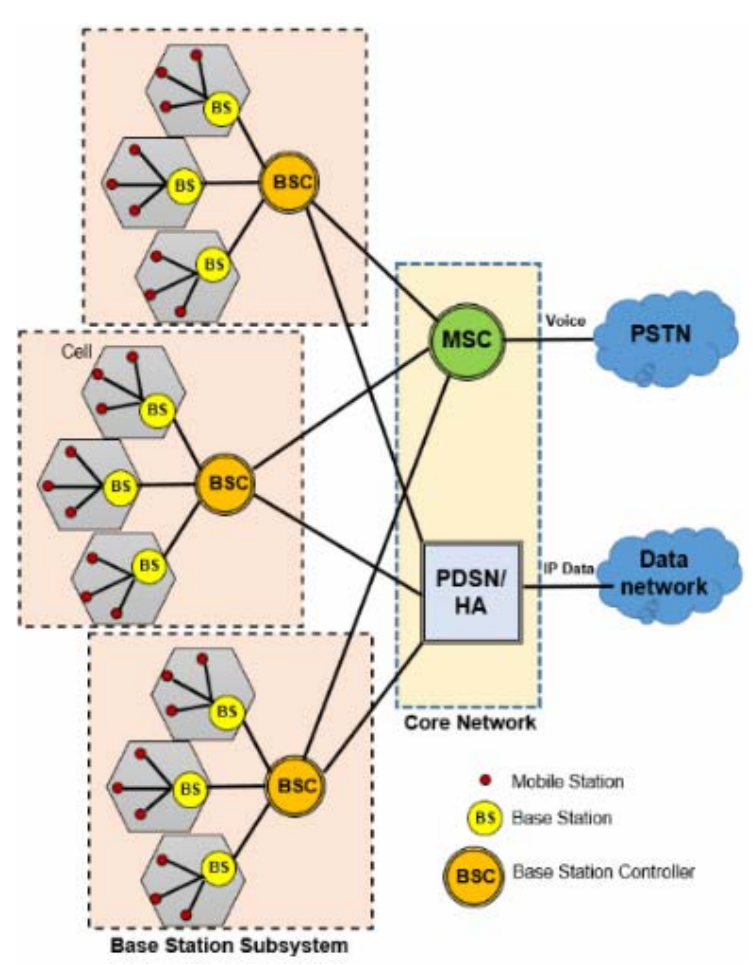

Figure 1. The NGWN infrastructure. 
Corresponding to the architectural building blocks of a wireless network, are three types of interconnects [2]. These are (1) mobile device to BS interconnect, which includes both forward and reverse radio links, (2) the BS to BSC interconnect, which is called the backhaul, and (3) BSC to MSC interconnect. The known hierarchical capacitated concentrator location problem, which is an extension of the concentrator location problem to multiple levels and a classical research issue in the telecommunications literature [3-6]. In [7], the authors studied the base station location and service assignment problem in a W-CDMA. A greedy strategy to optimal positioning of BSs for cellular radio networks and capacity planning of UMTS networks studied in [8-9]. A Tabu search and Genetic algorithm approach to cellular capacity expansion to maximizing the coverage area and minimizing the number of transmitters is presented in $[10,11]$. Yu et al in [12] proposed a set covering algorithm for given traffic and finding optimal solution configuration in a CDMA network. An alternate approach to capacity planning and expansion is introduced for 3G network system capacity without an increase in BSs using a cell splitting approach [13].

In latest papers [14-18], we have proposed a novel Particle Swarm Optimization (PSO), Ant Colony Optimization (ACO) and Genetic algorithms (GA) to optimal location of controllers in wireless networks and centralized wireless access network. In this paper, we focus on the Multi-Objectives Optimal of Upgrading Infrastructure (MOOUI) in NGWN and propose a new Genetic algorithm to solve it. The rest of this paper is organized as follows: Section 2 presents the MOOUI problem formulation. Section 3 presents our new algorithm to solve it based on GA algorithm. Section 4 is our simulation and analysis results, and finally, section 5 concludes the paper.

\section{Problemformulation}

In this section, we assume that network topology has $m$ mobile users, $n$ base stations, and $p$ base station controllers. we introduce the following notation in Table 1 below.

The MOOUI problem in NGWN has two steps the initial assignment of MSs to BS and the connection of BS to BSC and capacity expansion and traffic increase with constraint specifies that:

- $\quad$ Each mobile user $M S_{i}$ will be assigned to exactly one base station $B S_{j}$ of type $t$

- Mobile usersare within that base stations' maximum range MaxBS_Cov

- At most one base station of type $t$ can exist at location $j$

- if a base station $B S_{j}$ is operated, it has to be connected to a $B S C_{k}$ and the BSC has to be active.
Table 1. Notation defininition.

\begin{tabular}{|c|c|}
\hline Notation & Meaning \\
\hline$M$ & $\begin{array}{l}\text { Index set of Mobile user locations: } \\
M=\left\{M S_{i}, \forall i=1 . . m\right\}\end{array}$ \\
\hline$N$ & $\begin{array}{l}\text { Index set of all Base Station (BS): } \\
N=N 1 \cup N 2=\left\{B S_{j}, \forall j=1 . . n\right\} \\
N 1 \text { : Index set of existing BS; } \\
N 2 \text { : Index set of potential BS }\end{array}$ \\
\hline$P$ & $\begin{array}{l}\text { Index set of Base Station Controllers (BSC): } \\
P=P 1 \cup P 2=\left\{B S C_{k}, \forall k=1 . . p\right\} \\
P 1 \text { : Index set of existing BSC; } \\
P 2 \text { : Index set of potential BSC }\end{array}$ \\
\hline$T_{j}$ & Set of types available for $B S_{j}, \forall j \in N$ \\
\hline$S$ & $\begin{array}{l}\text { Set of commodity types: } \\
s= \begin{cases}1 & \text { if commondity type is voice } \\
2 & \text { if commondity type is data }\end{cases} \end{array}$ \\
\hline$N_{t}$ & Index set of all BS of type $t . N_{t}=N 1_{t} \cup N 2_{t}$ \\
\hline$D_{i}^{s}$ & $\begin{array}{l}\text { Demand of commodity type } s \text { for mobile user } \\
M S_{i}, \forall i \in M\end{array}$ \\
\hline$M a x B S_{-}$Cap $_{j_{t}}$ & Maximum capacity of $B S_{j}$ of type $t, \forall j \in N_{t}$. \\
\hline MaxBSC_Cap ${ }_{k}$ & Maximum capacity of $B S C_{k}, \forall k \in P$ \\
\hline$d_{i j_{t}}$ & $\begin{array}{l}\text { Distance of mobile user } M S_{i} \text { from } B S_{j} \text { of type } \\
t \forall i \in M, \forall j \in N_{t}\end{array}$ \\
\hline$M a x B S_{-} \operatorname{Cov}_{j_{t}}$ & Maximum coverage range for $B S_{j}$ of type $t$ \\
\hline $\begin{array}{c}\text { cost_connect }_{j_{t} k} \\
\text { cost_install } \\
k\end{array}$ & $\begin{array}{l}\text { Cost of connecting } B S_{j} \text { of type } t \text { to } B S C_{k} \\
\text { Cost of installing } B S C_{k}, \forall k \in P 2\end{array}$ \\
\hline $\begin{array}{l}\text { cost_upgrade }_{j} \\
\text { cost_setup }_{j_{t}}\end{array}$ & $\begin{array}{l}\text { Per channel cost of upgrading } B S_{j}, \forall j \in N 1 \\
\text { Cost of constructing and connecting } B S_{j}, \forall j \in N 2\end{array}$ \\
\hline
\end{tabular}

The capacity constraints of the model, in which we argue that BSs must have the necessary capacity to accommodate traffic demand of all demand types $\mathrm{s}$ for all MSs assigned to it and the BSC must have the necessary capacity to accommodate all BSs assigned to it.

In the first step, we use the indicator variables are:

$$
\begin{aligned}
\alpha_{j_{t}} & =\left\{\begin{array}{l}
1 \text { if } B S_{j} \text { of type } t \text { is operated } \\
0 \text { otherwise }
\end{array}\right. \\
\beta_{j_{t} k} & = \begin{cases}1 \text { if } B S_{j} \text { of type } t \text { is connected to } B S C_{k} \\
0 & \text { otherwise }\end{cases} \\
\delta_{k} & = \begin{cases}1 & \text { if } B S C_{k} \text { is operated in initial assigment } \\
0 & \text { otherwise }\end{cases}
\end{aligned}
$$

Figure 2 shows an example of an existing initial assignment that each mobile user can be assigned to only one BS, while each BS has to be connected to a single BSC. 


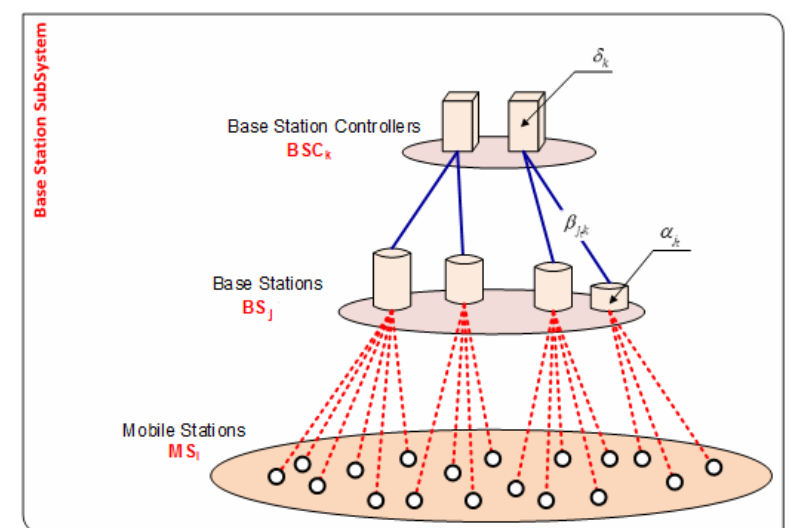

Figure 2. The indicator variables in Initial Assignment step.

In the second step, we use the decision variables are:

$$
\begin{aligned}
X_{i j_{t}} & = \begin{cases}1 & \text { if mobile user } M S_{i} \text { is connected to } B S_{j} \\
0 & \text { otherwise }\end{cases} \\
Y_{j_{t} k} & = \begin{cases}1 & \text { if } B S_{j} \text { of type } t \text { is connected to } B S C_{k} \\
0 & \text { otherwise }\end{cases} \\
Z_{j_{t}} & = \begin{cases}1 \text { if } B S_{j} \text { of type } t \text { is operated } \\
0 & \text { otherwise }\end{cases} \\
W_{k} & = \begin{cases}1 & \text { if } B S C_{k} \text { is operated } \\
0 & \text { otherwise }\end{cases}
\end{aligned}
$$

Figure 3 illustrates an assignment after capacity expansion and traffic increase, and indicates the respective decision variables. New wireless BSS infrastructure equipped with BS and BSC in red shades.

The objective of MOOUI is to minimize the total cost of expanding an initial wireless BSS to accommodate increased traffic demand.

The MOOUI problem can be defined as follows:

$$
\begin{aligned}
\operatorname{Min} & \left(\sum_{j=1}^{n} \sum_{k=1}^{p} \sum_{t \in T_{j}} \text { cost_connect }_{j_{t} k}\left(Y_{j_{t} k}-\beta_{j_{t} k}\right)\right. \\
& +\sum_{k \in P_{2}} \text { cost_install }_{k}\left(W_{k}-\delta_{k}\right) \\
& +\sum_{j \in N_{1}} \text { cost_upgrade }\left(\sum_{t \in T_{j}} \text { MaxBS_cap }_{j_{t}}\left(Z_{j_{t}}-\alpha_{j t}\right)\right) \\
& \left.+\sum_{j \in N_{2}} \sum_{t \in T_{j}} \text { cost_setup }_{j_{t}} Z_{j_{t}}\right)
\end{aligned}
$$

Subject to:

$$
\begin{aligned}
& \sum_{j=1}^{n} \sum_{t \in T_{j}} X_{i j_{t}}=1, \quad \forall i=\overline{1 . . m}
\end{aligned}
$$

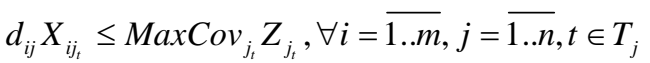

$$
\begin{aligned}
& \sum_{t \in T_{j}} Z_{j_{t}} \leq 1, \forall j=\overline{1 . . n}
\end{aligned}
$$

$$
\begin{gathered}
Z_{j_{t}} \leq \sum_{k=1}^{p} Y_{j_{t} k}, \forall j=\overline{1 . . n}, t \in T_{j} \\
Y_{j_{t} k} \leq W_{k}, \forall k=\overline{1 . . p}, j=\overline{1 . . n}, t \in T_{j} \\
\sum_{i=1}^{m} \sum_{s=1}^{2} D_{i}^{s} X_{i j_{t}} \leq M a x B S_{-} \operatorname{Cap}_{j_{t}} \times Z_{j_{t}}, \forall j=\overline{1 . . n}, t \in T_{j} \\
\sum_{j=1}^{n} \sum_{t \in T_{j}} Y_{j_{t} k} \leq M a x B S C_{-} C_{k a p_{k}} \times W_{k}, \forall k=\overline{1 . . p} \\
X_{i j_{t}} \in\{0,1\}, Y_{j_{t} k} \in\{0,1\}, Z_{j_{t}} \in\{0,1\}, W_{k} \in\{0,1\} \\
\forall i=\overline{1 . . m}, j=\overline{1 . . n}, k=\overline{1 . . p}, t \in T_{j}
\end{gathered}
$$

\section{A New Genetic Algorithm for the MOOUI}

\subsection{Represent and Decode an Individual}

In this section, we present a new genetic algorithm for the MOOUI problem with two populations $P O P_{X}$ and $P O P_{Y}$. The encoding of the $P O P_{X}$ configuration is by means of matrix $X=\left(x_{i j}\right)_{n \times m}, \quad(i=1 . . n, j=1 . . m)$ where $x_{i j}=1$ means that mobile user $M S_{i}$ has been connected to base station $B S_{j}$, and other wise, $x_{i j}=0$. The encoding of the $\mathrm{POP}_{\mathrm{Y}}$ configuration is by means of matrix

$$
Y=\left(y_{j k}\right)_{m p},(j=1 . . m, k=1 . . p),
$$

where $y_{j k}=1$ means that base station $B S_{j}$ has been connected to base station controller $B S C_{k}$, and other wise, $y_{j k}$ $=0$.

\subsection{Initialization}

We use fully random initialization in order to initialize the individuals $P O P_{X}$ ensure that the individual $x$ satisfies constraints in (9)(10)(14) and (16). Each individual $x$, we fully random initialization in order to initialize the individuals $P O P_{Y}$ ensure that the individual $y$ satisfies constraints in (12)(13)(15) and (16).

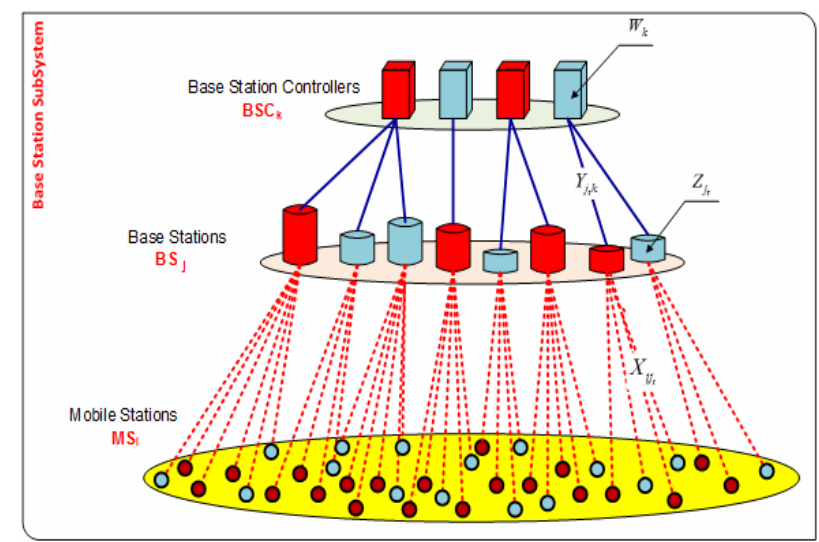

Figure 3. The assignment after capacity expansion and traffic increase with decision variables. 


\subsection{Crossover Operator}

This operator minics the mating process in the nature. To do crossover in $P O P_{X}$, two individuals are picked first and two integer numbers $(i, j)$ (crossover point is $x_{i j}$ ) are generated randomly between $[1, n]$ and $[1, m]$ (where nis number of MSs and $m$ is number of BSs).

Then the offspring is generated by interchanging the second halves of its parent, as illustrated in Figure 4.

In the crossover stage, the algorithm examines all pairs of individuals. It begins with the pairs that include the individual with a higher fitness value until the population size becomes twice of the original size. Similar, we apply crossover operator to $\mathrm{POP}_{\mathrm{Y}}$.

\subsection{Mutation Operator}

The mutation operation is one kind of random change in the individual of $P O P_{X}$. In our algorithm, point wise mutation is adopted, in which one gene in the individual is changed with a certain probability, referred to as the mutation probability. This operator allows the algorithm to search for new and more feasible individuals in new corners of the solution spaces.

To do mutation, an individual is randomly selected from the BS and the selected BS is called the mutation point, as illustrated in Figure 5.
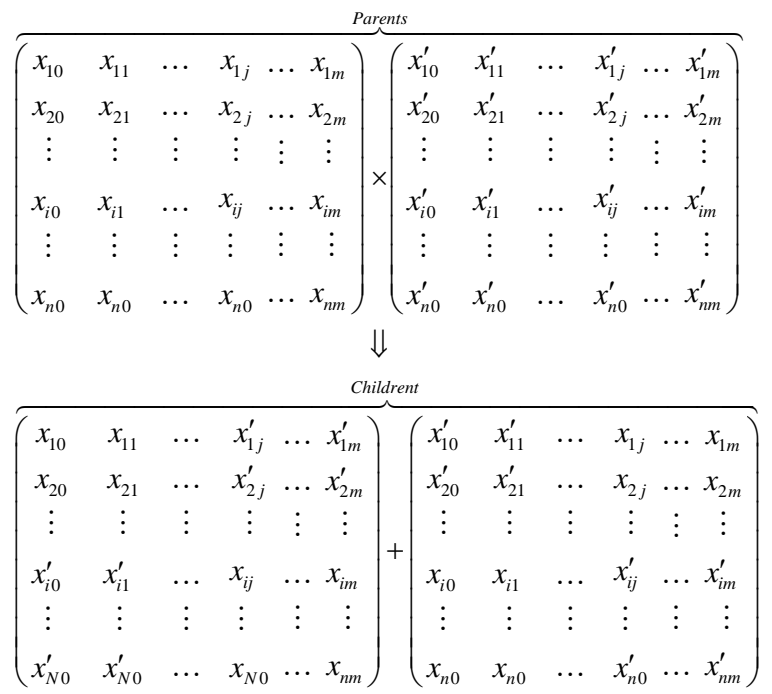

Figure 4. The crossover operator for population $P O P_{x}$.

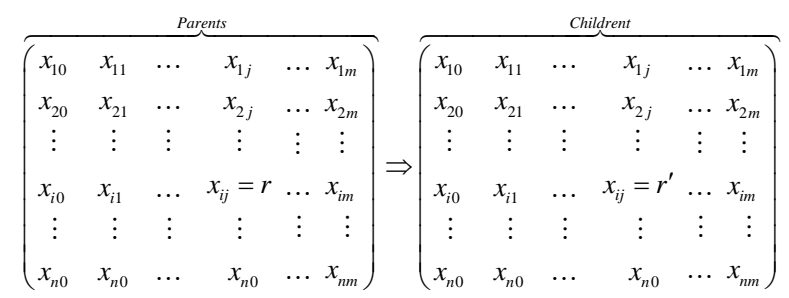

Figure 5. The mutation operator for population $P O P_{x}$.
The mutation stage is implemented until either population size becomes twice of the original size or all individuals in the current generation are examined. Similar, we apply mutation operator to $P O P_{Y}$.

\subsection{Evaluation Function}

After the mutation, each solution

$$
s=\left\{(x, y) \mid x \in \operatorname{POP}_{X}, y \in \operatorname{POP}_{Y}\right\}
$$

is satisfies constraints in (9)-(16). The cost function of solution $s$ computed by formula (8).

\subsection{Our GA Algorithm Proposed}

The pseudo-code of our algorithm as follows:

A NEW GENETIC ALGORITHM FOR THE MOOUI
BEGIN
INITIALISE population $P O P_{X}$ withrandomcandidatesolutions;
REPEAT
1. SELECT parents in $P O P_{X}$;
2. RECOMBINE pairsofparents in $P O P_{X}$;
3. CROSSOVER the resultingoffspring in $P O P_{X} ;$
4. MUTATION the resultingoffspring in $P O P_{X} ;$
5. FOR each eachcandidate $x \in P O P_{X}$ DO
5.1 INITIALISE population $P O P_{Y}$ withrandomcandidate
$\quad$ solutions $y \in P O P_{Y}$ follows candidate $x \in P O P_{X}$;
5.2. SELECT parents in $P O P_{Y}$;
5.3. RECOMBINE pairsofparents in $P O P_{Y} ;$
5.4. CROSSOVER the resultingoffspring in $P O P_{Y} ;$
5.5. MUTATION the resultingoffspring in $P O P_{Y} ;$
5.6. COMBINE Solution
$\quad s=\left\{(x, y) \mid x \in P O P_{X}, y \in P O P_{Y}\right\}$
6. EVALUATE FUNCTION newsolutions s by formula $(8) ;$
7. SELECT individualsxfor the nextgeneration;
UNTIL (TERMINATION $C O N D I C T I O N$ issatisfied)
END

\section{Experiments and Results}

\subsection{The Problems Tackled}

In our experiments, we have tackled several MOOUI instances of different difficulty levels. There are 10 MOOUI instances with values for $\mathrm{M}, \mathrm{N}$ and $\mathrm{P}$ is shown in Table 2.

\subsection{Parametersfor the GA Algorithm}

In our experiments, we have already defined our crossover probability as 0.7 , we will work with a population size of 500 and a mutation rate of $p_{m}=1 / \mathrm{m}$.

Our GA algorithm to tackle these problems can be specified as below in Table 3 . 
Table 2. Main characteristic of the problems tackled.

\begin{tabular}{|c|c|c|c|c|c|c|c|c|c|}
\hline \multirow[t]{2}{*}{$\begin{array}{c}\text { Problem } \\
\#\end{array}$} & \multirow[t]{2}{*}{$\begin{array}{l}\text { Mobile Users } \\
\text { (M) }\end{array}$} & \multicolumn{4}{|c|}{ Base Stations } & \multicolumn{4}{|c|}{ Base Stations Controllers } \\
\hline & & $\mathbf{N}$ & N1 & N2 & Types & $\mathbf{P}$ & P1 & $\mathbf{P} 2$ & Types \\
\hline 1. & 10 & 4 & 3 & 1 & 1 & 3 & 2 & 1 & 1 \\
\hline 2. & 30 & 6 & 3 & 3 & 3 & 4 & 2 & 2 & 3 \\
\hline 3. & 100 & 10 & 6 & 4 & 4 & 5 & 2 & 3 & 3 \\
\hline 4. & 250 & 25 & 15 & 10 & 5 & 15 & 10 & 5 & 5 \\
\hline 5. & 500 & 50 & 30 & 20 & 8 & 20 & 10 & 10 & 6 \\
\hline 6. & 1000 & 150 & 90 & 60 & 10 & 50 & 30 & 20 & 8 \\
\hline 7. & 2500 & 350 & 200 & 150 & 20 & 100 & 40 & 60 & 10 \\
\hline 8. & 5000 & 550 & 350 & 200 & 30 & 150 & 100 & 50 & 15 \\
\hline 9. & 7500 & 750 & 450 & 300 & 40 & 200 & 150 & 50 & 20 \\
\hline 10. & 10000 & 950 & 650 & 300 & 50 & 300 & 200 & 100 & 30 \\
\hline
\end{tabular}

Table 3. The GA Algorithm Specifications.

\begin{tabular}{cl}
\hline Representation & \multicolumn{1}{c}{ Matrix $X=\left(x_{i j}\right)_{n \times m}, Y=\left(y_{j k}\right)_{m \times p}$} \\
\hline Recombination & One point crossover \\
Recombination probability & $70 \%$ \\
Mutation & Each value inverted with independent probability $p_{m}$ per position \\
\hline Mutation probability & $p_{m}=1 / m$ \\
Parent selection & Best out of random two \\
Survival selection & Generational \\
Population size & $P O P_{X}=P O P_{Y}=500$ \\
Number of offspring & 500 \\
Initialization & Random \\
Termination condition & No improvement in last 100 generations \\
\hline
\end{tabular}

\subsection{Numerical Analysis}

We evaluate the performance of our algorithms to optimize of capacity expansion with multi-objectives. The experiment was conducted on Genuine Intel ${ }^{\circledR}$ CPU Duo Core $3.0 \mathrm{GHz}, 2 \mathrm{~GB}$ of RAM machine. We ran experiment $\mathrm{GA}$ algorithm implemented using $\mathrm{C}$ language.

Comparing values of objective function between initial solution and optimal solution shown in Figure 6 with Population size $P O P_{X}=P O P_{Y}=500$ and termination condition is 100 generations. Figure 7 shown time processing of MOOUI instances tackle. The results show that problems with the small number of $M, N, P$ such as problem \#1, \#2, \#3, \#4 and \#5, algorithm has approximate optimal results fast with small interactions. However, when the problem size is large, the optimal results may be slower such as problem \#6, \#7, \#8, \#9 and \#10. Convergence speed is not the same and depends on the distribution of parameters data.

Figure 8(a) shows an existing initial assignment of problem \#2. In which, three types of base station are (BS1, BS6), (BS2, BS3), (BS4, BS5); BS2, BS3 BSC2, BSC4 are existing BSCs; BSC1, BSC3 are potential BSCs; BS3, BS4, BS6 areexisting BSs; BS1, BS2, BS5 arepotential BSs. MS6, MS16, MS22, MS29 are not 
connected. Figure 8(b) shows an optimal solution with BSC2 is replaced by BSC3, BS4 is replaced by BS2 and BS5. BS1 is added and connect to BSC4. Red edges are replace connections and black edges are existing connections.

To evaluation the effect of population size and number of iterations to the value of the objective function. We consider the problem \#2 with the iterations can vary from 100 to 500 and fixed population size is 500 , comparing results are shown in Figures 9(a) and (b). To comparing the problem \#2 with the population size can vary from 500 to 1000 and fixed iterations size is 100 , comparing results are shown in Figures 10(a) and (b). The experimental results show that the algorithm can be implemented with the big number of interactions and large population size in polynomial time. From this result, we confirmed that this is a promising approach to solve this problem.

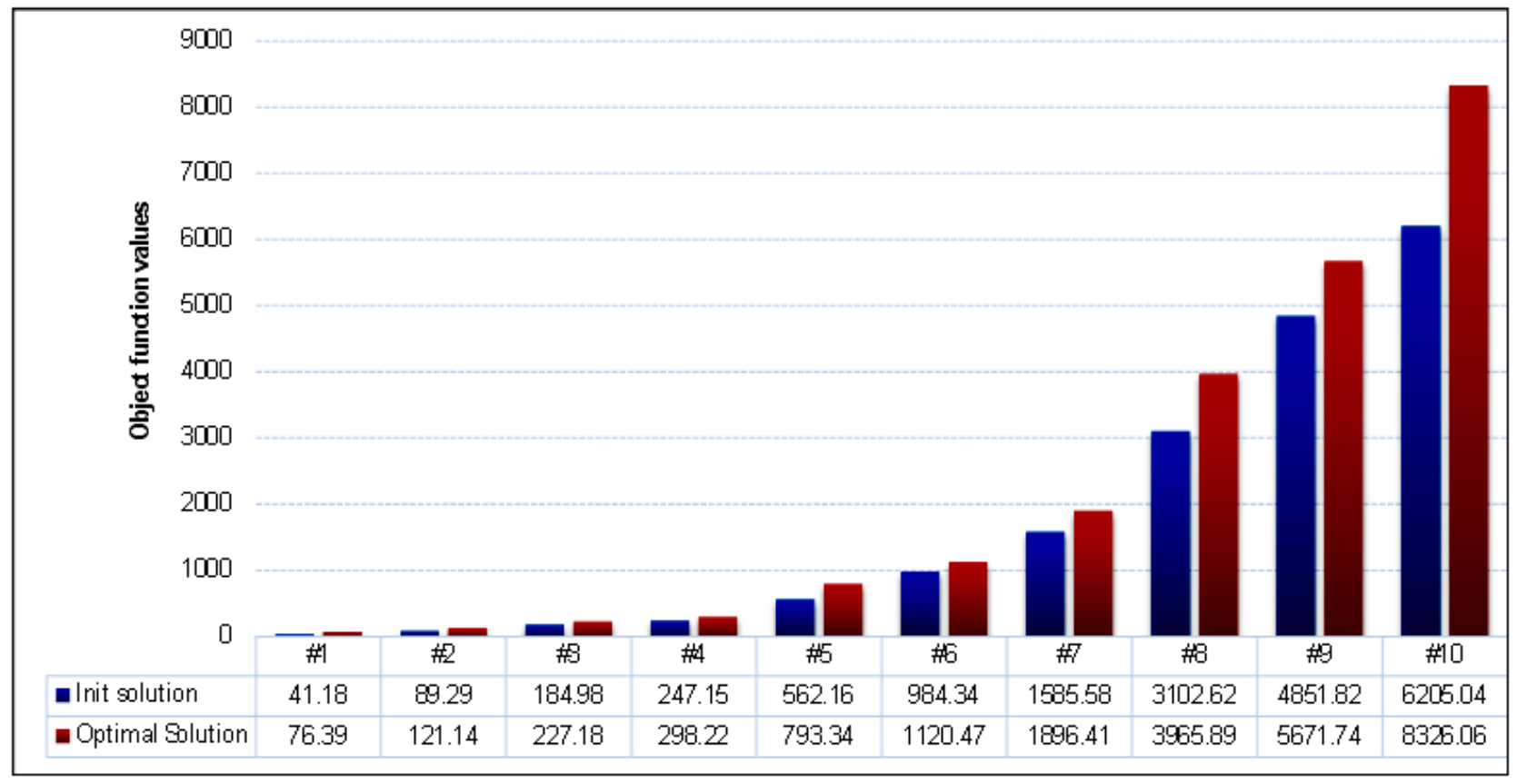

Figure 6. Comparing value of capacity expansion in the MOOUI instances tackle.

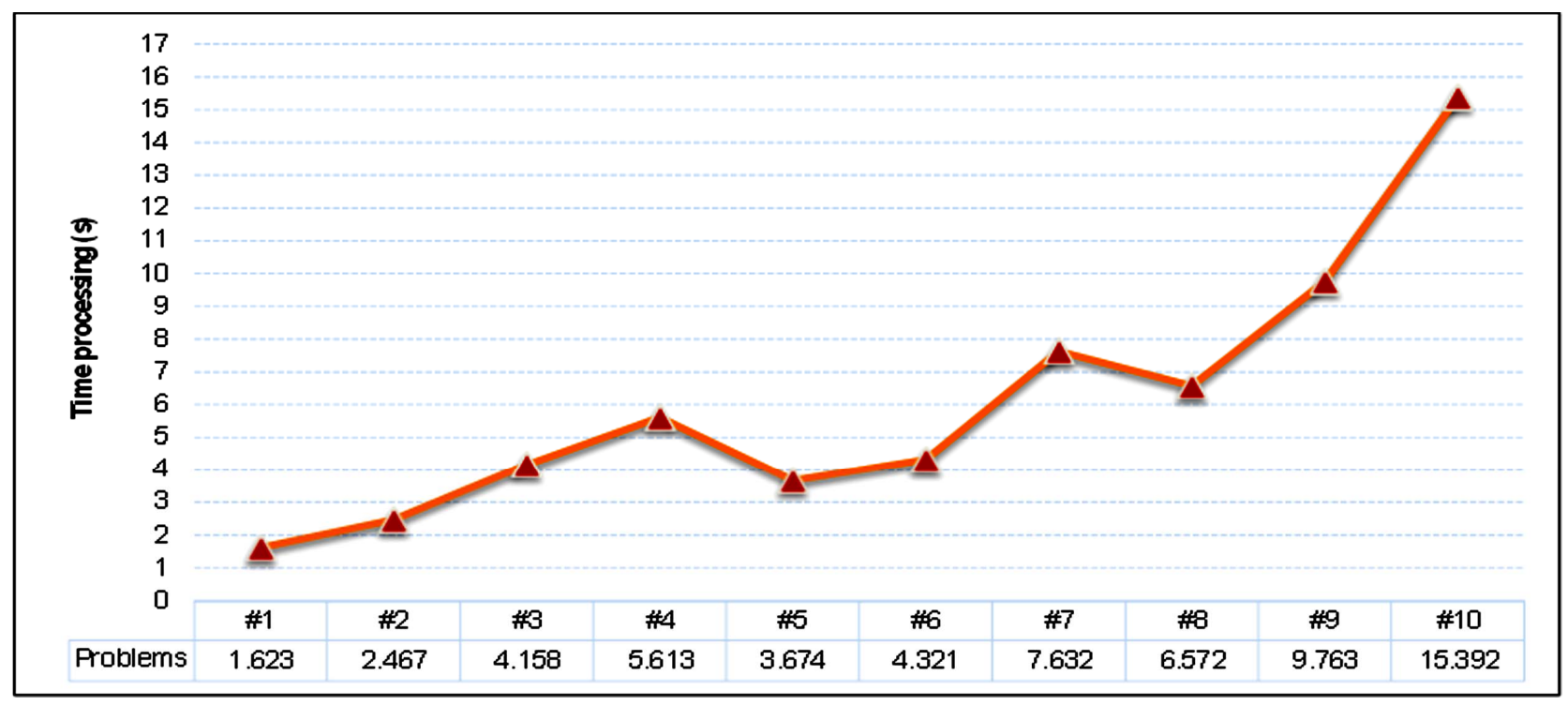

Figure 7. Time processing MOOUI instances tackle. 


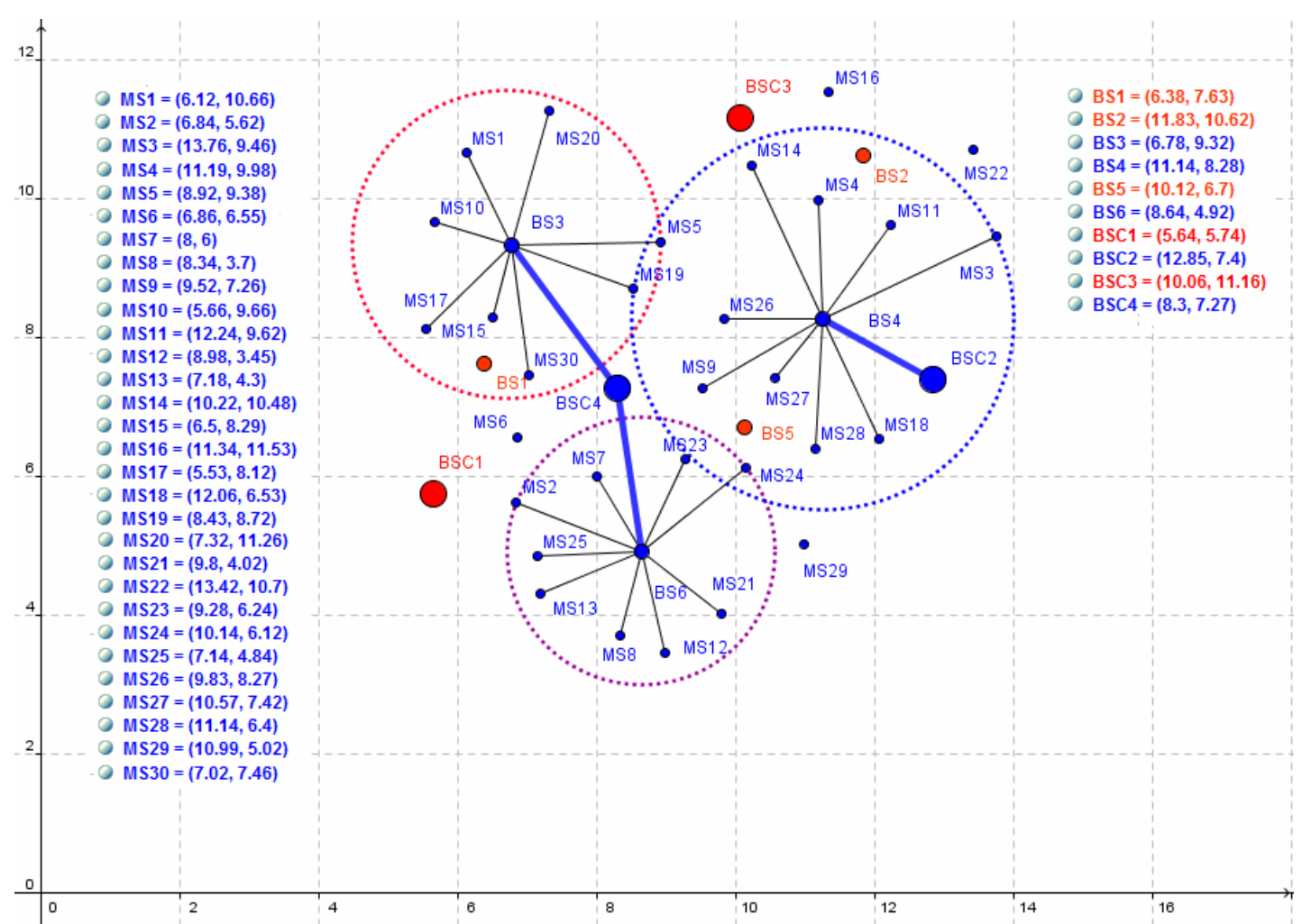

(a)

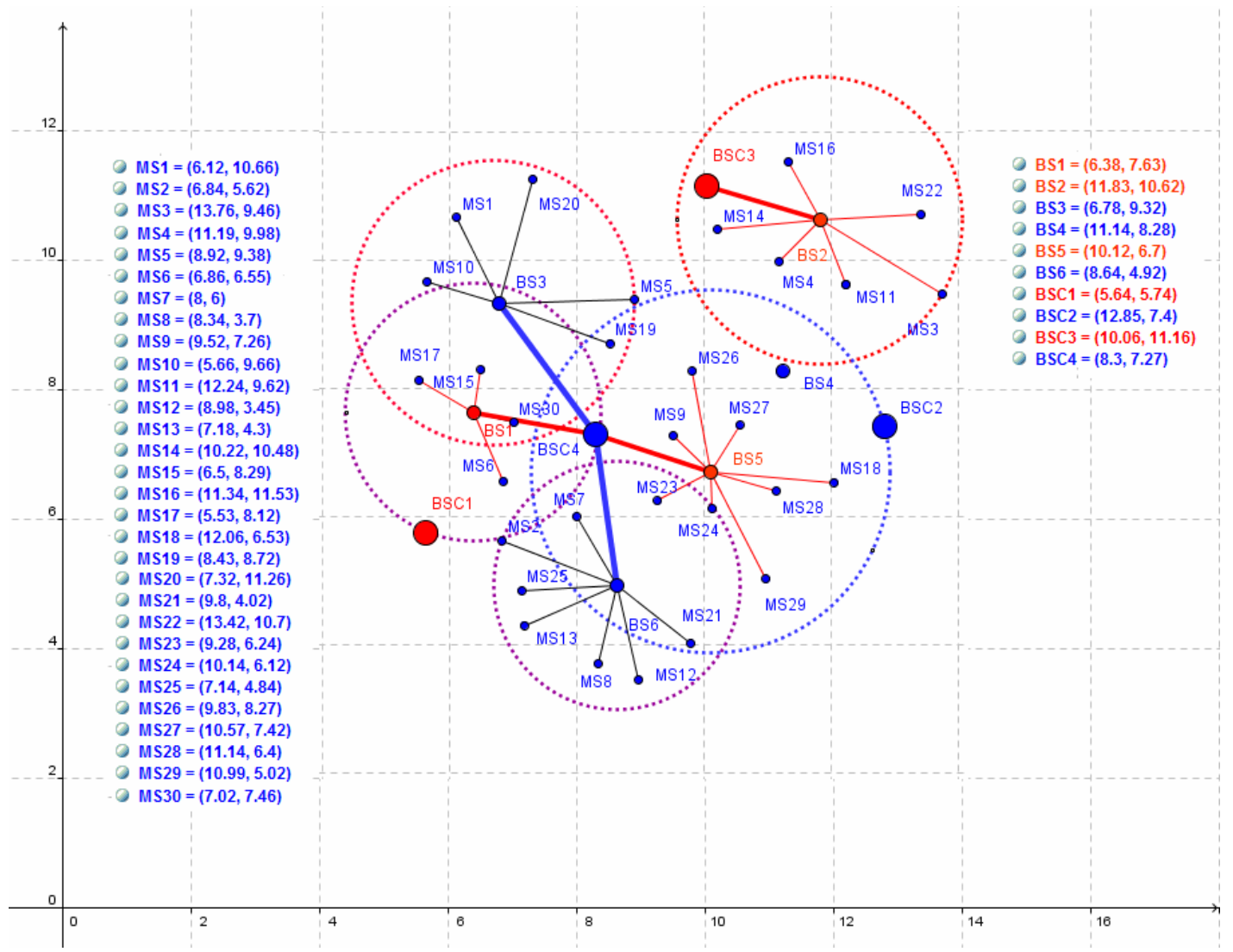

(b)

Figure 8. (a) An existing initial assignment of problem \#2; (b) An optimal capacity expansion of problem \#2. 


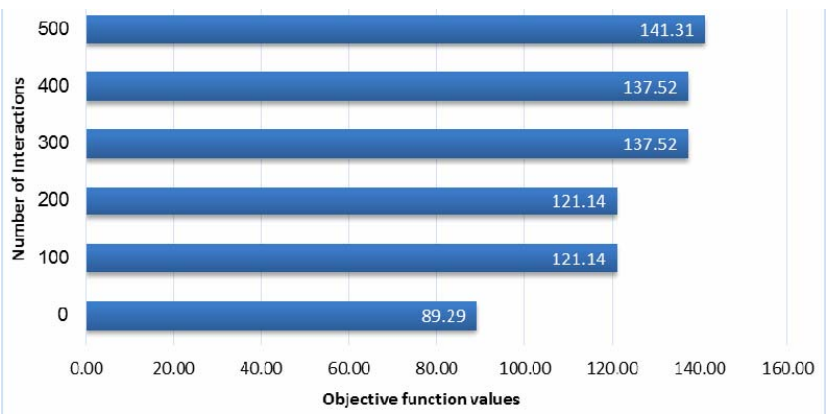

(a)

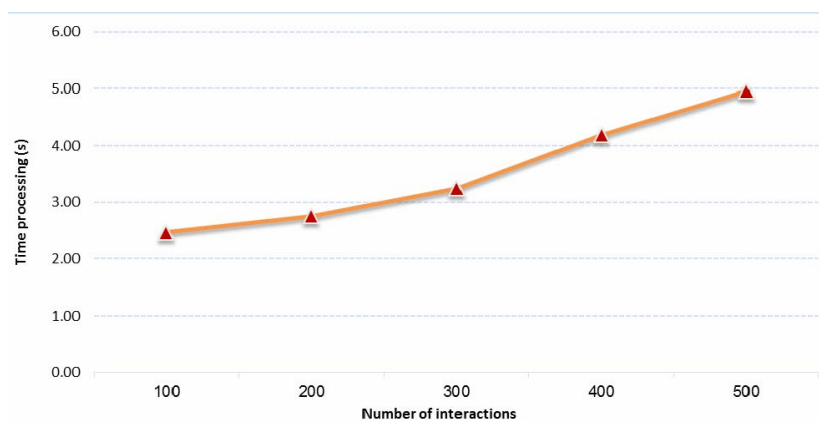

(b)

Figure 9. (a) Comparing objective function values of problem\#2 with different number of interactions; (b) Comparing time processing of problem\#2 with different number of interactions.

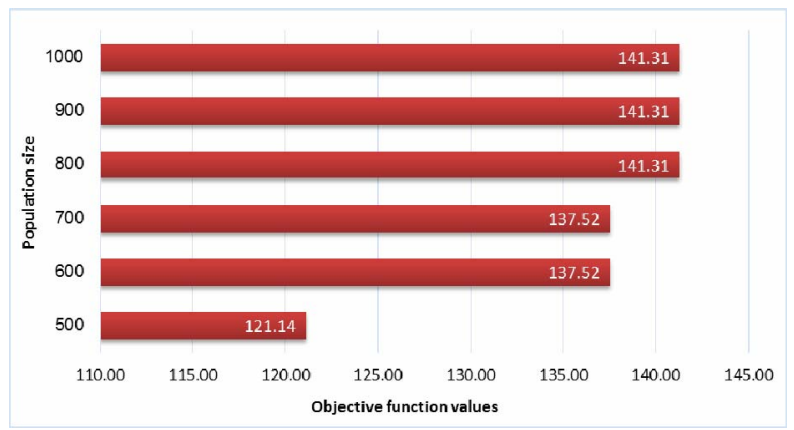

(a)

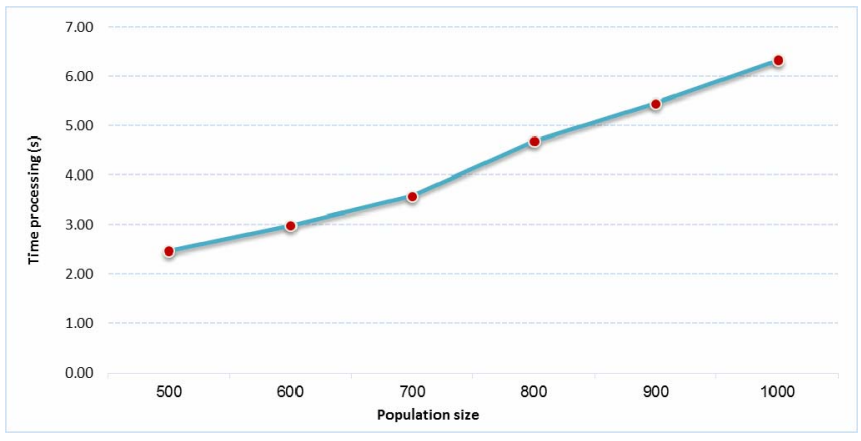

(b)

Figure 10. (a) Comparing objective function values of problem\#2 with different population size; (b) Comparing time processing of problem \#3 with different population size.

\section{Conclusions}

In this paper, we propose a new genetic algorithm has double population to solve Multi-Objectives Optimal of Upgrading Infrastructure problem in NGWN. Our network topology model has two levels in which mobile users are sources and both base stations and base station controllers are concentrators. The objective function is the sources to concentrators connectivity cost as well as the cost of the installation, connection, replacement, and capacity upgrade of infrastructure equipment. We generate two populations satisfies constraints and combine to build solutions and evaluate the performance of our algorithm with data randomly generated. Numerical results show that our algorithms is a promising approach to solve this problem.

\section{Acknowledgements}

This research is partly supported by the QG.12.21 project of Vietnam National University, Hanoi.

\section{REFERENCES}

[1] Commworks, Wireless Data for Everyone. http://www.commworks.com. Technical Paper, 3Com
Corporation, 2001.

[2] Siemens Mobile. UMTS. http://www.siemens.de. White Paper, 2001.

[3] Mirzaian, A. and K. Steiglitz. A Note on the Complexity of the Star-Star Concentrator Problem. IEEE Transactions On Communications. No. 29, 1981, pp. 1549-1552. doi:10.1109/TCOM.1981.1094884

[4] B. Gavish, A System for Routing and Capacity Assignment in Computer Communication Networks. IEEE Transactions of Communications, No. 37, 1989, pp. 360-366. doi:10.1109/26.20116

[5] S. Narasimhan and H. Pirkul, "The Hierarchical Concentrator Location Problem,” Computer Communications, Vol. 15, No. 3, 1992, pp. 185-191. doi:10.1016/0140-3664(92)90079-T

[6] R. Gupta and J. Kalvenes, "Hierarchical Cellular Network Design with Channel Allocation," In Proceedings of the Ninth Annual Workshop on Information Technologies \& Systems, 1999, pp. 155-160.

[7] Kalvenes, J., J. Kennington and E. Olinick. Base Station Location and Service Assignment in W-CDMA Networks. Technical Report 02-EMS-03. SMU, 2002.

[8] Mathar R. and T. Niessen. Optimum positioning of base stations for cellular radio networks. Wireless Networks. Vol. 6, No. 6. 2000, pp. 421-428. doi:10.1023/A:1019263308849

[9] R. Mathar and M. Schmeink, Capacity Planning of UMTS 
Networks. In Proceedings of Sixth INFORMS Telecommunications Conference, Boca Raton, Florida 2002.

[10] C. Y. Lee and H. Kang, “Cell Planning with Capacity Expansion in Mobile Communications: A Tabu Search Approach," IEEE Transactions on Vehicular Technology, Vol. 49, No. 5. 2000, pp. 1678-1691. doi:10.1109/25.892573/

[11] P. Calegari, F. Guidee, P. Kuonen and D. Wagner, "Genetic Approach to Radio Network Optimization for Mobile Systems,” IEEE VTC, pp. 755-759, 1997.

[12] C. Yu, S. Subramanian and N. Jain, "CDMA cell site optimization using a set covering algorithm," In Proceedings of Eight Int. Network Planning Symposium, 1998, pp. 75-78.

[13] R. Giuliano, F. Mazzenga and F. Vatalaro, "Smart cell sectorization for third generation CDMA systems," Wireless Communications and Mobile Computing, Vol. 2, No. 3, 2002, pp. 253-267.

[14] Dac-Nhuong Le, "Genetic Algorithm Applied to the Optimal Centralized Wireless Access Network," International Journal of Information \& Network Security (IJINS), Vol. 2, No. 2, 2013, pp. 129-137.

[15] Dac-Nhuong Le and Nhu Gia Nguyen, “A New Evolutionary Approach for Gateway Placement in Wireless Mesh Networks," International Journal of Computer Networks and Wireless Communications (IJCNWC), Vol. 2, No. 5, 2012, pp. 550-555.
[16] Dac-Nhuong Le, "PSO and ACO Algorithms Applied to optimal Resource Allocation to Support QoS Requirements in Next Generation Networks," International Journal of Information \& Network Security (IJINS), Vol. 2, No. 3, 2013, pp. 216-228.

[17] Dac-Nhuong Le, "PSO and ACO Algorithms Applied to Optimizing Location of Controllers in Wireless Networks," International Journal of Computer Science and Telecommunications (IJCST), Vol. 3, No. 10, 2012, pp. 1-7.

[18] Dac-Nhuong Le, "Optimizing the cMTS to Improve Quality of Service in Next Generation Networks based on ACO Algorithm," International Journal of Computer Network and Information Security (IJCNIS), Vol. 5, No. 4, 2013, pp. 25-30. doi:10.5815/ijcnis.2013.04.04

[19] Dac-Nhuong Le, "EA and ACO Algorithms Applied to Optimizing Location of Controllers in Wireless Networks," International Journal of Network Communication and Networking (IJNCN), Vol. 3, No. 2, 2013, pp. 17-27.

[20] Dac-Nhuong Le, Nhu Gia Nguyen and Vinh Trong Le, "A Novel Ant Colony Optimization-based Algorithm for the Optimal Centralized Wireless Access Network," Lecture Notes of the Institute for Computer Sciences, Social Informatics and Telecommunications Engineering (LNICST), Springer 2013. 